\title{
N132D: A CHEMICAL AND DYNAMIC ANALYSIS
}

\author{
RALPH S. SUTHERLAND, M.A. DOPITA \\ Mount Stromlo and Siding Spring Observatories \\ The Australian National University \\ Canberra \\ Australia
}

\begin{abstract}
We have obtained 2D spectrophotometry of the LMC supernova remnant N132D, achieving a higher spatial resolution than previous studies (Lasker 1978, 1980). A model based on a simple expanding ring of oxygen rich material is shown to be inadequate. We find incomplete arcs of shocked material with individual components of differing compositions. Oxygen rich material appears as rapidly moving small knots in parts of these arcs and in a larger diffuse cloud, all in the central 6-10 pc region of the remnant. We propose a model for this remnant involving the interaction of the supernova blastwave with an incomplete ring of normal composition material to produce reflected shockwaves in the inner regions of the remnant, exciting oxygen rich material.

In the knots of more normal composition a wide range of shock conditions prevail. Apart from 'normal' fully ionized radiative shocks with velocities $100-200 \mathrm{~km} / \mathrm{s}$ the very steep Balmer decrements in the filaments of some spectra are consistent with partially ionized, slower, shocks. Other spectra show very strong emission lines for the higher ionization species, OIII and NeIII, which could be explained in terms of either strong shocks with long recombination timescales or as very young shocks that have not yet achieved steady flow.
\end{abstract}

\section{Introduction}

This presentation represents the initial results of a spectrophotometric study of the supernova remnant (SNR) N132D, in the Large Magellanic cloud. This SNR is one of a small group of remnants known to display shocked emission from material that is composed of almost purc oxygen. This material presumably originates from the progenitor star itself and so gives us an opportunity to examine some of the properties of supernova progenitor stars. It has been suggested (Van den Bergh 1988 and references therein) that the oxygen rich class of SNR can bc produced by the explosion of a Wolf-Rayet star in a type Ib event. The models suggest that the main shockwave propagates out through the evacuated bubble produced by the WR stcllar wind, and that a reverse shockwave propagates back into ejected material, exciting oxygen rich matcrial.

The data used here for this initial analysis came from observations totalling $16 \times 1500$ second integrations using the double beam spectrograph on the ANU 2.3m ATT at Siding Spring in December 1989. A resolution of $\sim 1.5 \AA$ /pixel was achieved over a range $3300 \AA$ to $8000 \AA$ and the spectrograph slit was moved in $2.5 "$ steps, maintaining a north-south orientation. The data was calibrated using white dwarf flux standards, and the reduction was carried out using the IRAF package.

Supplementary images using narrow band filters for $\mathrm{H} \alpha$ and [OIII] were taken in May 1990) using the AAT 3.9m telescope and the 1024×1024 pixel Thompson CCD.

\section{Results}

The results are presented here in the form of diagrams and images. Detailed measurements and analysis will be presented in a future publication. 


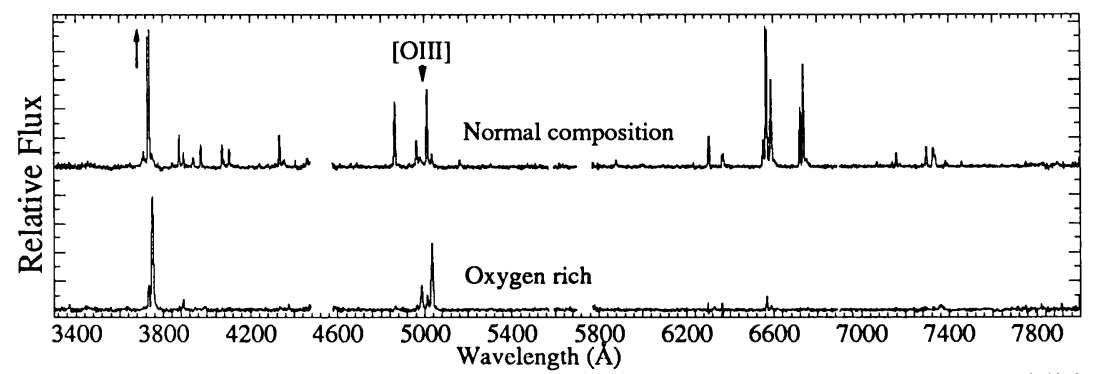

Figure 1. Spectra obtained for two immediately adjacent knots of similar [OIII] brightness.

N132D Radial Velocity map. [OII] $3729 \AA$ synthetic narrow band images.

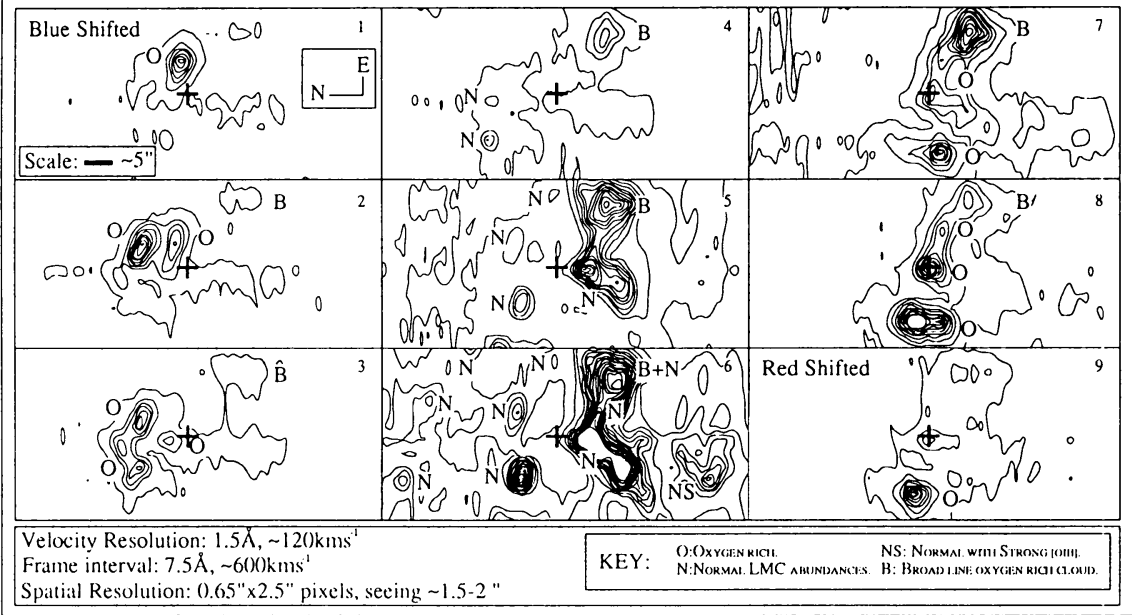

Figure 2. Radial velocity map of the inner regions of N132D. Compositions of some parts are marked. The + is close to the mark by Mathewson et al 1983. Continuum sources have been subtracted.
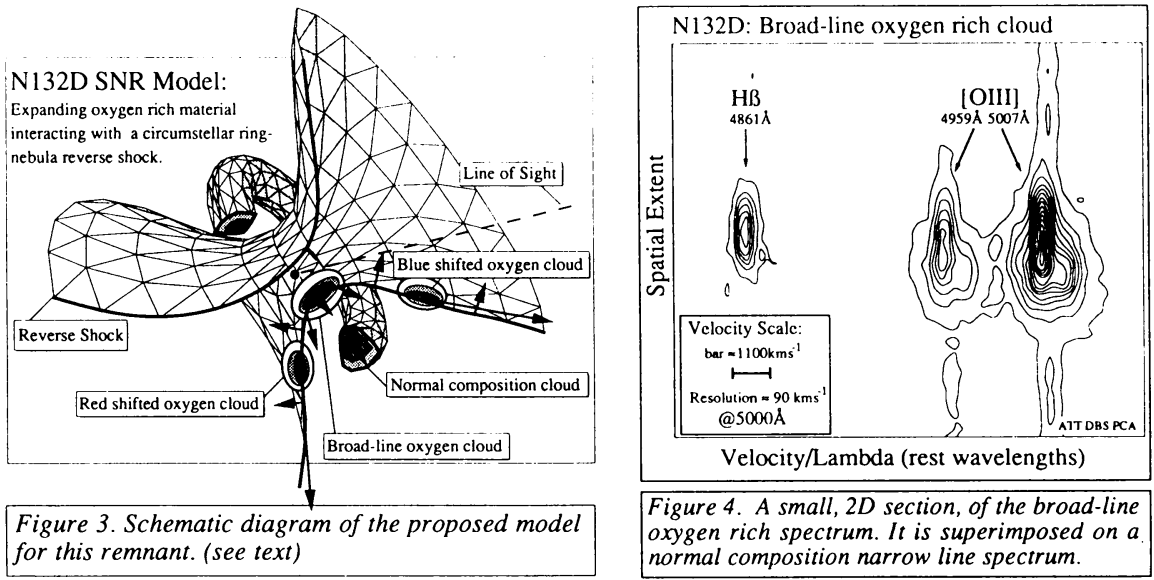

Figure 4. A small, $2 D$ section, of the broad-line oxygen rich spectrum. It is superimposed on a normal composition narrow line spectrum. 
We find that the oxygen rich rings described by Lasker (1980) and Mathewson et al. (1983) are heterogeneous in composition and composed largely of non-oxygen rich material. These 'normal' knots naturally appear in [OIII] images because they do contain oxygen (figure 1).

The oxygen rich knots have a wide range of velocities, while the normal material is less exireme in its velocity range. (figure 2). The velocity map is only partially convincing in showing an expanding ring geometry. The broad-line oxygen cloud is completely incompatible with a simple expanding ring geometry. (figure 4)

Finally, There appear to be a wide range of shock conditions in this remnant, in terms of composition, in shock velocities and possibly shock ages.

\section{Discussion}

Detailed shock modelling will be required to determine meaningful chemical analyses of this remnant. However, early analysis indicates that invoking local circumstellar reddening, suggested by Danziger and Leibowitz (1985), may not be necessary to explain steep Balmer decrements. A slow shock propagating into a partially ionized medium can display very high $\mathrm{H} \alpha: \mathrm{H} \beta$ ratios.

With these observations we can now propose a model for the inner emission regions of SNR N132D that incorporates an interaction with the local circumstellar environment as follows.

A dense, but not necessarily complete, ring of normal composition material forms during the WR evolution phase of the star, similar to material seen around other WR stars (Chu 1981). The supernova blastwave then propagates out and interacts with the ring components (figure 3 ). The reverse shockwave for the SNR could then form an equatorially pinched geometry. The reverse shocks from the ring in the inner parts of the remnant are thus responsible for the excitation of the inner oxygen rich material.

Given this geometry of an inclined ring producing a reverse shock, the patchy but spherically distributed oxygen rich material interacting with this shock wave would produce the high velocity spectra observed. One prediction of this model is that if a large cloud of oxygen were to straddle the ring material then a whole range of velocities for the one cloud should be observed. This is precisely the case for the newly discovered broad-line cloud that overlays some normal composition material (figure 4).

This model is still compatible with the low density ISM cavity model (Hughes 1987), requircd to bring a homogeneous Sedov model age of $\sim 4000-7000 \mathrm{yr}$, (based on X-ray observations) in line with the $1300 \mathrm{yr}$ age proposed by Lasker (1980, based on the optical). The low cavity density limits may be able to be relaxed somewhat now that different scale heights have been introduced.

This makes N132D a WR analogue of the type II SN models by Itoh and Fabian (1984) once the differences in the material distribution are accounted for.

\section{References}

Chu, Y-H. (1981), Ap. J. 249, 195.

Danziger, I.J. and Leibowitz, E.M. (1985), M.N.R.A.S. 216, 365.

Hughes, J.P. (1987), Ap. J. 314, 103.

Itoh, H., Fabian A.C. (1984), M.N.R.A.S. 208, 645.

Lasker, B.M. (1978), Ap. J. 223, 109.

Lasker, B.M. (1980), Ap. J. 237, 765.

Mathewson, D.S., Ford, V.L., Dopita, M.A., Tuohy, I.R., Long, K.S. and Helfand, D.J. (1983), Ap. J. Suppl Ser. 51, 345.

van den Bergh, S. (1988), Ap. J. 327, 156. 